\title{
MODELAMIENTO MOLECULAR \\ DE LA DERMASEPTINA SP2 \\ EXTRAÍDA DE Agalychnis spurrelli
}

\author{
MOLECULAR MODELING OF DERMASEPTINE SP2 \\ EXTRACTED FROM Agalychnis spurrelli
}

\section{Sebastián Cuesta H. ${ }^{1}$, Josefa Arias de P. ${ }^{2}$, Felipe Gallegos P. ${ }^{2}$, Carolina Proaño B. , Ailín Blasco-Zúñiga ${ }^{4}$, Miryam Rivera I. ${ }^{4}$ \& Lorena Meneses $\mathrm{O}^{2}$.}

Recibido: 15 octubre 2018/ Aceptado: 12 noviembre 2018

DOI: 10.26807/ia.v7i1.95

Palabras claves: Acoplamiento molecular, Agalychnis spurrelli, dermaseptinas, péptidos antimicrobianos.

Keywords: Agalychnis spurrelli, antimicrobial peptides,

Dermaseptins, molecular docking.

\section{RESUMEN}

En esta investigación se presenta un estudio computacional de la dermaseptina SP2 (DRS-SP2) extraída de exudado de la piel de la rana Agalychnis spurrelli.

1 Laboratorios LIFE, Quito, Ecuador (sebastian_cuesta@yahoo.com )

2 Pontificia Universidad Católica del Ecuador, Facultad de Ciencias Exactas y Naturales, Escuela de Cien cias Químicas, Laboratorio de Química Computacional, Quito, Ecuador (emiarias_10coc@hotmail.com; edfelipe28@gmail.com; Immeneses@puce.edu.ec)

3 Universidad Regional Amazónica IKIAM, Tena, Ecuador (carolina.proano@ikiam.edu.ec)

4 Pontificia Universidad Católica del Ecuador, Facultad de Ciencias Exactas y Naturales, Escuela de Ciencias Biológicas, Laboratorio de Investigación de Citogenética y Biomoléculas de Anfibios, Centro de Investigaciones para la Salud en América Latina (CISeAL) Quito, Ecuador (ablasco@puce.edu.ec; mriverai@puce.edu.ec) 
Ensayos experimentales han permitido extraer, purificar y obtener la secuencia de aminoácidos de este péptido, además de demostrar sus propiedades antimicrobianas contra Escherichia coli, Staphylococcus aureus y Candida albicans. Con la secuencia dilucidada, se realizó un estudio computacional de la estructura obteniéndose sus propie-dades físico-químicas, su estructura secundaria y su similitud con otros péptidos conocidos. Además, se realizó el acoplamiento molecular de este péptido conla membrana celular y varias enzimas conocidas para suprimir a estos micro-organismos. Los resultados muestran que la DRS-SP2 es un péptido catiónico $\alpha$-helicoidal con un punto isoeléctrico de 10,68 y carga positiva +3 a pH fisio-lógico. Se determinó que su estructura es diferente a todas las dermaseptinasque se encuentran en bases de datos llegando a un porcentaje de identidadmáximo del $80 \%$. Estudios de acoplamiento molecular sugieren que el meca- nismo de acción de este péptido no se da por la inhibición de vías enzimáticas vitales para el microorganismo, sino por lisis celular.

\section{ABSTRACT}

In this research, we present a computational study of Dermaseptine SP2 (DRSSP2) extracted from the skin of the frog Agalychnis spurrelli. Experimental assays allowed extracting, purifying and obtaining the amino acid sequence of this peptide. Furthermore, they have demonstrated its antimicrobial properties against Escherichia coli, Staphylococcus aureus and Candida albicans. With the sequence, a computationalstudy of the structure was carried out, obtaining its physical-chemical properties, its secondary structure and its similarity with other known peptides. Also, a mo-lecular docking study of this peptide was performed against cell membrane and several enzymes known to kill these microorganisms. Results showed that DRS-SP2 is an $\alpha$-helical cationic peptide with an isoelectric point of 10.68 and apositive charge +3 at physiological $\mathrm{pH}$. It was determined that its structure is different to all dermaseptines found in databases reaching a maximum identity of $80 \%$. Molecular docking studies suggest the mechanism of action of thispeptide is not given by the inhibition of a vital enzymatic pathway, but by destruction of the microorganism by cell lysis. 


\section{INTRODUCCIÓN}

Las glándulas granulares en la piel de algunas especies de ranas, sintetizan péptidos con una actividad antimicrobiana de amplio espectro contra bacterias, hongos y protozoos, por lo que constituyen parte esencial del sistema inmune innato del animal (Conlon, 2012; Holthausen et al., 2017; Lacombe et al.,2015; Manzo et al., 2014; Scorciapino et al., 2013).

Hasta la fecha, más de 900 péptidos eucariotas han sido descritos, de los cuales más de la mitad han sido aislados de ranas de la familia Hylidae de Sudamérica o Europa, y ranas de la familia Ranidae proveniente de Asia o Nor- teamérica (Nicolas \& Amri, 2009; Ni-colas \& Ladram, 2013; Samgina et al., 2012).

Gran parte de estos péptidos pertenecen a la familia de las dermaseptinas. Las dermaseptinas son una familia de péptidos aislados principalmente del género de ranas Phyllomedusa (Nicolas \& Ladram, 2013). Estos son péptidos anfipáticos policatiónicos (ricos en lisina) y $\alpha$-helicoidales, con 28-34 residuos de longitud, que se encuen- tran entre los principales constituyentes de las secreciones de la piel de las ranas arbóreas (Ammar et al., 1998; Melchiorri \& Negri, 2009).

Estudios han demostrado su actividad contra virus, células cancerígenas, propiedades antioxidantes, antitumorales, antidiabetes, inhibición de enzimas, y efectos quimiostáticos, por lo que son interesantes para el descubrimiento de nuevos medicamentos (Holthausen et al., 2017; Huang et al., 2017; Marani et al., 2015). Al ser miembros prototípicos de una gran clase de péptidos catiónicos, estos dañan la membrana, al experimentar una transición de espiral a hélice, al unirse a las bicapas lipídicas (Nicolas \& Amri, 2009; Nicolas \& Ladram, 2013).

A concentraciones micromolares, estos péptidos causan la lisis celular de una amplia gama de microorganismos, que incluyen bacterias Grampositivas y Gram-negativas, levaduras, protozoos y hongos, sin efectos nocivos contra las células de mamíferos (Ammar et al., 1998; Nicolas \& Amri, 2009; Nicolas \& Ladram, 2013). 
Análisis espectroscópicos y computacionales, sugieren que la flexibilidad estructural del terminal $\mathrm{N}$ y $\mathrm{C}$ juegan un papel crucial en la interacción con la membrana peptídica (Stutz et al., 2017).

Agalychnis spurrelli, comúnmente llamada rana mono planeadora, es una especie de anfibio del orden Anura y de la familia Hylidae. Esta especie habita en el Ecuador en las provincias de Esmeraldas, Los Ríos, Manabí, Carchi y Pichincha es nocturna y viven en bosques húmedos, cerca de cuerpos de agua, donde se re - producen (Duellman, 2001; Gray, 1997, MECN, 2010; Ortega-Andrade, 2008).

El objetivo del presente estudio fue aplicar técnicas computacionales en la dermaseptina SP2 extraída de la piel de la rana Agalychnis spurrelli, para determinar las propiedades físico-químicas, la estructura secundaria y la similitud con otros péptidosconocidos. Además, se realizó el acoplamiento molecular de este péptido con la membrana celular y varias enzimas conocidas por matar a cepas de Escherechia coli, Staphylococcus aureus y Candida albicans.

\section{MATERIALES Y MÉTODOS}

Para esta investigación, se utilizaron diferentes técnicas computacionales que incluyen el uso de distintos programas, servidores y páginas web. Las propiedades físico-químicas de la dermaseptina SP2 (DRS-SP2), fueron predichas utilizando las páginas ExPasy (Gasteiger et al., 2005), Pepcalc (Innovagen, 2015), Biosyn (Bio-Synthesis Inc, 2018), JPred 4 (Drozdetskiy et al., 2015), PSIPred (Jones, 1999). Las comparaciones del péptido en estudio con otros péptidos de diferentes especies de anfibios, fue- ron realizadas mediante la base de datos Uniprot (https://www.uniprot. org/). La estructura tridimensional del péptido fue obtenida a través del programa Pymol (Schrödinger, 2017) y la optimización de la estructura se realizó usando mecánica estadística con el campo de fuerza MM2, implementado en el "software" ChemDraw (PerkinElmer Informatics, 2009). Una vez obtenido el péptido optimizado, se procedió a realizar el estudio de acoplamiento molecular (Docking) mediante el programa Autodock 
VINA (Trott \& Olson, 2010). Además, se realizó la interacción de este péptido con los fosfolípidos de la mem- brana celular mamífera y bacteriana para obtener mayor información sobre su mecanismo de acción.

\section{RESULTADOS}

Mediante el uso de Cromatografía Líquida de alta eficiencia (HPLC) para la separación y técnicas de clonaje y secuenciación, se obtuvo la secuencia de aminoácidos de DRS-SP2 (ASWKVFLKNIGKAAGKAVLNSVT
DMVNQ). Este péptido fue sintetizado y probado experimentalmente, siendo efectivo frente a tres microorganismos E.Coli, S.Aureus y C.Albicans (Tabla 1).

Tabla 1. Resultados experimentales de la bioactividad de la DRS-SP2

\begin{tabular}{|c|c|c|c|c|c|c|}
\hline Nombre & Origen & Fuente & Microorganismo & Cepa & Bioactividad & MIC* \\
\hline & & & Echerichia coli & $\begin{array}{l}\text { ATCC } \\
25922 \\
\end{array}$ & $\mathrm{Si}$ & 8 \\
\hline \multirow[t]{2}{*}{ DRS-SP2 } & sintético & $\begin{array}{l}\text { Agalychnis } \\
\text { spurrelli }\end{array}$ & $\begin{array}{c}\text { Staphylococcus } \\
\text { aureus }\end{array}$ & $\begin{array}{l}\text { ATCC } \\
25923\end{array}$ & $\mathrm{Si}$ & 8 \\
\hline & & & $\begin{array}{l}\text { Candida } \\
\text { albicans }\end{array}$ & $\begin{array}{l}\text { Aislado } \\
\text { clínico }\end{array}$ & $\mathrm{Si}$ & 32 \\
\hline
\end{tabular}

* Concentración inhibitoria mínima (mg/L)

Una vez comprobada la actividad antimicrobiana in vitro, se procedió a estudiar el péptido mediante técnicas computacionales. En primer lugar, se predijeron las propiedades físico-químicas de la DRS-SP2 que se muestran en la Tabla 2. La carga neta del péptido en dependencia del $\mathrm{pH}$ y la flexibilidad del mismo se muestran en la Figura 1. 
Tabla 2. Propiedades físico-químicas de la DRS-SP2 aislada de Agalychnis spurrelli

\begin{tabular}{ll}
\hline Secuencia & ASWKVFLKNIGKAAGKAVLNSVTDMVNQ \\
\hline Número de aminoácidos & 28 \\
\hline Peso molecular & 2990,51 \\
\hline Fórmula molecular & $\mathrm{C}_{134} \mathrm{H}_{221} \mathrm{~N}_{37} \mathrm{O}_{38} \mathrm{~S}_{1}$ \\
\hline Número de átomos & 431 \\
\hline Punto isoeléctico & 10,64 \\
\hline Aminoácidos con carga positiva & 4 \\
\hline Aminoácidos con carga negativa & 1 \\
\hline \% de aminoácidos básicos & 14,29 \\
\hline \% de aminoácidos neutros & 32,14 \\
\hline \% de aminoácidos ácidos & 3,57 \\
\hline \% de aminoácidos hidrofóbicos & 50,00 \\
\hline Carga & +3 (básico) \\
\hline Carga neta a pH 7 & 3 \\
\hline Hidrofobicidad a pH 6.8 & 27.07 \\
\hline Coeficiente de extinción & $5500 \mathrm{M}^{-1} \mathrm{~cm}{ }^{-1}$ a 280 nm \\
\hline Vida media estimada & 4,4 horas (reticulocito mamífero, in vitro) \\
\hline Índice de inestabilidad & $>20$ horas (levadura, in vivo) \\
\hline
\end{tabular}

En cuanto a la estructura secundaria, tanto JPred, PSIpred como la estructura optimizada, predijeron que este péptido va a tener una estructura secundaria en forma de hélice alfa ( $\mathrm{Fi}$ gura 2).

La DRS-SP2 fue comparada con péptidos de la misma familia en especies diferentes, para determinar su porcentaje de similitud (Tabla 3). La similitud de la DRS-SP2 con diferentes dermaseptinas va de un 29,63\% con la dermaseptina 8 de Phyllomedusa tarsius a un $82,14 \%$ con la derma septina 2 de la misma especie.

La interacción del péptido con las diferentes enzimas muestra que, en ningún caso, la DRS-SP2 va a tener una mayor afinidad con la enzima en comparación con un inhibidor conocido (Tabla 4). 


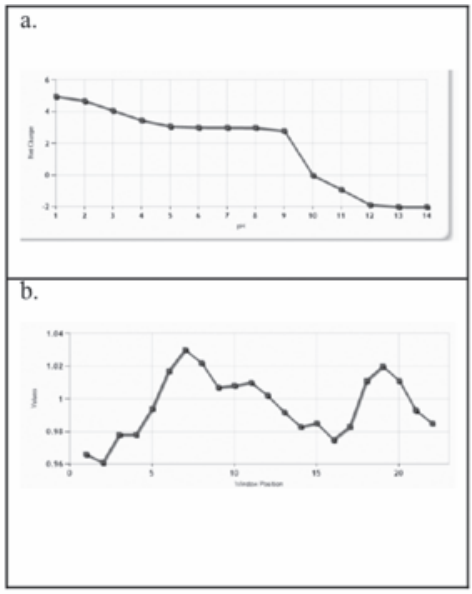

a.

b.

Figura 1. Propiedades de la DRS-SP2. a. Carga neta en función del pH. b. Flexibilidad del péptido en función de la secuencia aminoácida (Bio-Synthesis Inc, 2018)

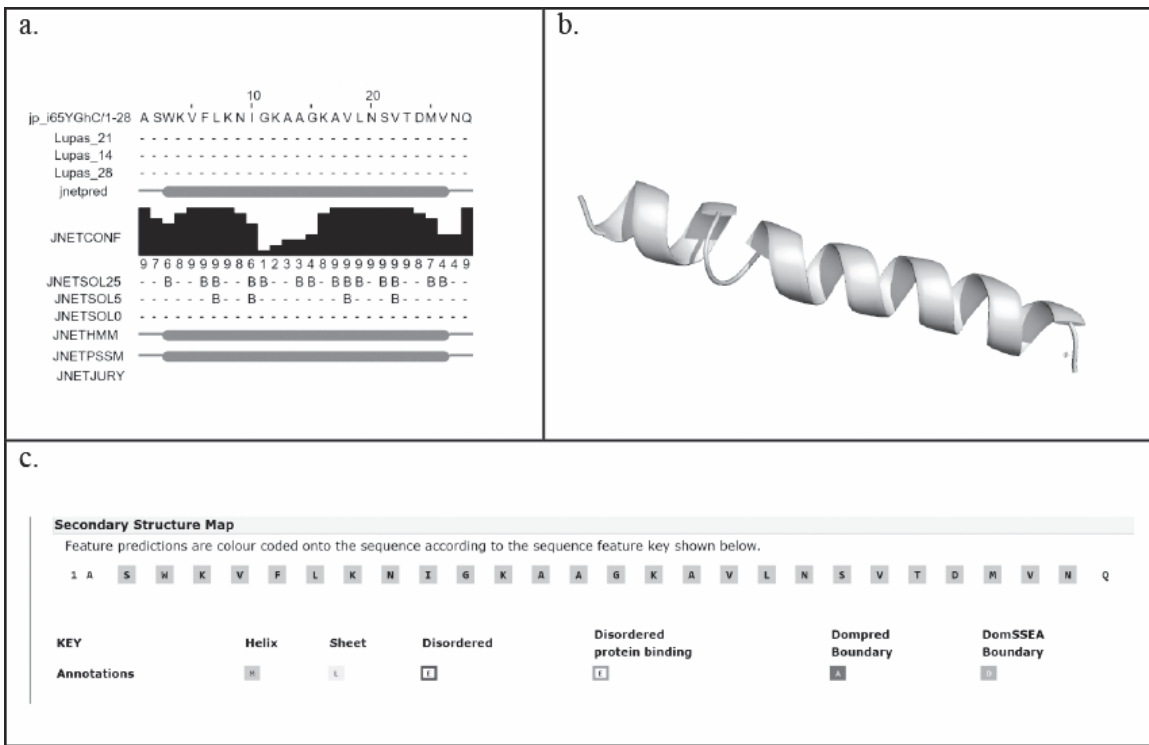

Figura 2. Estuctura secundaria de la DRS-SP2. a. Predicción obtenida de JPred. b. Optimización del péptido con el campo de fuerza MM2.

c. Predicción obtenida de PSIpred 
Tabla 3. Comparación de varias Dermaseptinas con DRS-SP2

\begin{tabular}{lccccc}
\hline Entrada & Nombre & Organismo & Secuencia & Longitud & $\begin{array}{c}\% \text { ident. } \\
\text { DRS-SP2 }\end{array}$ \\
\hline P84936 & Dermaseptin-H6 & Phyllomedusa azurea & GLWSTIKQKG KEAAIAAAKA AGQAALGAL & 29 & 38.10 \\
\hline P80280 & Dermaseptin-4 & Phyllomedusa sauvagei & ALWMTLLKKV LKAAAKALNA VLVGANA & 27 & 44.44 \\
\hline P83637 & Dermaseptin-01 & Phyllomedusa oreades & GLWSTIKQKG KEAAIAAAKA AGQAALGAL & 29 & 38.10 \\
\hline P84922 & Dermaseptin-2 & Phyllomedusa tarsius & ALWKDILKNV GKAAGKAVLN TVTDMVNQ & 28 & 82.14 \\
\hline P84600 & Dermaseptin-5 & $\begin{array}{c}\text { Phyllomedusa } \\
\text { hypochondrialis }\end{array}$ & GLWSTIKQKG KEAAIAAAKA AGQAALGAL & 29 & 38.10 \\
\hline P84921 & Dermaseptin-1 & Phyllomedusa tarsius & GLWSKIKETG KEAAKAAGKA ALNKIAEAV & 29 & 44.00 \\
\hline P84925 & Dermaseptin-5 & Phyllomedusa tarsius & GLWSKIKEAA KTAGKAAMGF VNEMV & 25 & 37.50 \\
\hline P84927 & Dermaseptin-7 & Phyllomedusa tarsius & ALWKDVLKKI GTVALHAGKA ALGAVADTI Q & 31 & 53.57 \\
\hline C0HKP8 & Dermaseptin-4 & Phyllomedusa & GLWSTIKQKG KEAAIAAAKA AGKAALNAASEAL & 33 & 40.00 \\
\hline P84928 & Dermaseptin-8 & Phyllomedusa tarsius & SLRGFLKGVG TALAGVGKVV ADQFDKLLQAGQ & 32 & 29.63 \\
\hline
\end{tabular}

Tabla 4. Valores de Afinidad ente la DRS-SP2 y diferentes enzimas

\begin{tabular}{lcccc}
\hline Organismo & Enzima & Inhibidor conocido & $\begin{array}{c}\text { Afinidad (kcal/mol) } \\
\text { Inhibidor conocido }\end{array}$ & DRS-SP2 \\
\hline S. aureus & $\begin{array}{c}\text { Proteína de unión } \\
\text { de Penicilina G-acil 2A }\end{array}$ & Ceftobiprole & $-9,5$ & 5,1 \\
\hline \multirow{2}{*}{ E. coli } & Hidrolasa AmiA & Muramil tetrapeptideo & $-7,1$ & $-5,0$ \\
\hline & ADN girasa B & ADP & $-10,4$ & $-4,2$ \\
\hline C. albicans & $\begin{array}{c}\text { Proteína de unión de } \\
\text { Penicilina Transglicosilasa 1b }\end{array}$ & Moenomicina & $-7,3$ & $-5,1$ \\
\hline & Exo-B-(1,3)-glucanasea & Castanospermina & $-7,0$ & $-5,3$ \\
\hline & Proteasa aspártica & A70* & $-7,7$ & $-6,1$ \\
\hline
\end{tabular}

* A70: N-etil-N-[(4-metilpiperazin-1-il)carbonil]-D-fenilalanil-N-[(1S,2S,4R)-4-(butilcarbamoil)-1-(ciclohexilmetil)2-hidroxi-5-metilhexil]-L-norleucinamida

El acoplamiento molecular entre la DRS-SP2 y las membranas celulares, tanto de células de mamífero como de bacteria, muestra una mayor afini- dad de este péptido hacia la membrana de célula bacteriana en 0,8 $\mathrm{kcal} / \mathrm{mol}$ (Tabla 5). 
Observando gráficamente la interacción (Figura 3), se puede ver que el péptido penetra por la membrana de la célula bacteriana, cosa que no ocurre en la membrana de la célula mamífera.

a.

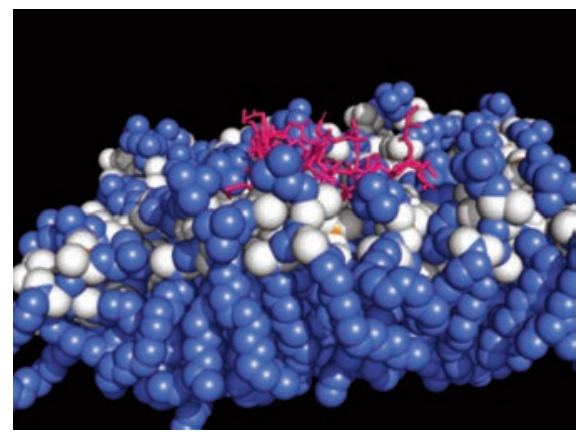

Tabla 5. Interacción entre la membrana celular y la DRS-SP2

\begin{tabular}{cc}
\hline Membrana celular & Afinidad (kcal/mol) \\
\hline Mamífera & $-3,2$ \\
\hline Bacteriana & $-4,0$ \\
\hline
\end{tabular}

b.

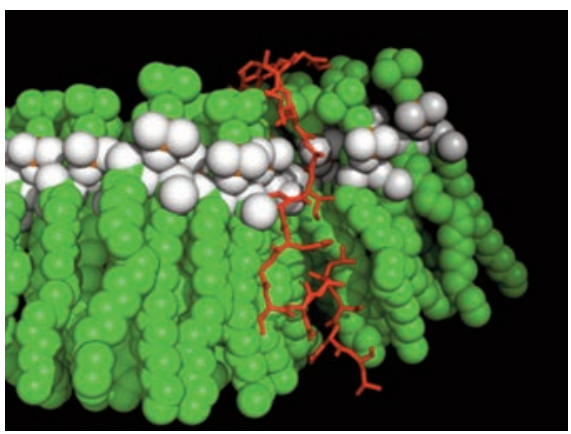

Figura 3. Interacción de la DRS-SP2 con: a. Membrana celular mamífera; b. Membrana celular bacteriana

\section{DISCUSIÓN}

Los resultados experimentales muestran que la DRS-SP2 extraída de la secreción de la piel de la Agalychnis spurrelli, posee propiedades antimicrobianas contra bacterias Gram negativas (E. coli), bacterias anaero bias Gram positivas (S. aureus) y hongos (C.albicans).

Dentro de sus propiedades, la DRSSP2 es un péptido de cadena corta
(28 aminoácidos) que posee en su estructura cuatro lisinas (aminoácidos básicos) y solo un aminoácido negativo (ácido aspártico); esto le confiere una característica básica reflejada en su punto isoeléctrico $(10,64)$ y su carga neta a pH 7 (3).

El coeficiente de extinción se define como la fuerza que tiene una sustancia, en este caso el péptido, de absor- 
ber luz a determinada longitud de onda por concentración molar (Gill et al., 1989). En este caso, la longitud de onda estudiada fue $280 \mathrm{~nm}$. Esta medida está íntimamente relacionada con la composición del péptido, ya que sólo ciertos aminoácidos, los que poseen un anillo aromático en su estructura, son capaces de absorber radiación electromagnética a esta longitud (tirosina, triptófano y fenilalanina), el triptófano es el que más absorbe. En el caso de la DRS-SP2, dos aminoácidos son los responsables de este coeficiente de extinción, un triptófano y una fenilalanina.

La vida media predicha para este péptido es de 4,4 horas en reticulocitos mamíferos, y de más de 10 horas en E. coli. Esto permite, de manera preliminar, inferir que esta dermaseptina podría en un futuro ser usada en humanos para combatir infecciones microbianas, debido a que la vida media en humanos es menor que en una bacteria.

El índice de inestabilidad estima la estabilidad de una proteína en un tubo de ensayo, basado en un estudio estadístico en dipéptidos en solución (Guruprasad et al., 1990). El valor ob- tenido de 7,48 indica que el péptido es estable, lo cual era esperado ya que, en la naturaleza, este necesita ser excretado a través de la piel de la rana, entrar en contacto con la bacteria y ejercer su función.

La predicción de la flexibilidad de la DRS-SP2, muestra que las zonas de los extremos y una pequeña zona en el centro del péptido son las partes menos flexibles de la proteína, mientras que dos zonas intermedias entre estas son las más flexibles. Esto no era esperado, ya que en solución, las partes que deberían tener una mayor flexibilidad son los extremos. Un estudio más detallado mediante técnicas de dinámica molecular sería necesario para poder corroborar o descartar esta predicción obtenida.

En cuanto a la estructura secundaria, las tres predicciones utilizadas muestran al péptido como una hélice alfa, tal como se indica en la literatura (Melchiorri \& Negri, 2009). Existe una pequeña diferencia ente los tres métodos, donde, JPred predice que el péptido se presenta en forma de hélice alfa en toda su estructura, con excepción de los dos primeros y los dos últimos aminoácidos. PSIpred, en 
cambio, muestra una estructura alfa helicoidal en todo el péptido, a excepción del primer y el último aminoácido. Finalmente, la estructura optimizada es un péptido alfa-helicoidal en toda la secuencia, a excepción del primer aminoácido, los dos últimos y los aminoácidos 9 y 10 (Asparagina e Isoleucina).

Con la base de datos de Uniprot, se compararon cientos de dermaseptinas descubiertas en otras especies de ranas, con la obtenida a partir de Agalychnis spurrelli. Los porcentajes de similitud encontrados muestran que este péptido, aislado de la rana mono planeadora, posee una estructura secundaria y una longitud similar a las encontradas en otras especies de ranas, al demostrar que el péptido pertenece a la misma familia de las dermaseptinas y compartir sus propiedades antimicrobianas. La mayor similitud se encontró con la dermaseptina 2 de la rana tarsio (Phyllomedusa tarsius), que también habita en territorio ecuatoriano. Sería interesante investigar a nivel genético cual es la relación que existe entre estas dos especies y como esto determina la similitud en sus péptidos de defensa microbiana.
El estudio de modelación molecular mostró que el mecanismo de acción de la DRS-SP2 no se da por inhibición de ninguna de las vías enzimáticas propuestas. En el caso de la Proteína de unión de Penicilina G-acil 2A, uno de los inhibidores conocidos es el Ceftobiprole. Este medicamento pertenece a la familia de las cefalosporinas (Wishart et al., 2018). En las interacciones enzima-sustrato, la carga y el tamaño del inhibidor es de suma importancia. En el caso de este antibiótico, su carga en $\mathrm{pH}$ fisiológico es - 1 en comparación a +3 del DRS$\mathrm{SP} 2$. Además, en su estructura, el antibiótico posee solo 58 átomos en comparación a los 431 átomos del péptido, lo cual va a hacer que no logre entrar en el bolsillo enzimático, y sí logra entrar parcialmente, la diferencia de cargas afecte la unión (Figura 4).

En el caso de la Hidrolasa AmiA, tanto el inhibidor (Muramil tetapéptido) como el DRS-SP2 son péptidos básicos con diferencia en su longitud (4 aminoácidos versus 28) y en la separación que existe entre los aminoácidos básicos. En el caso del Muramil tetapéptido, al estar más seguidos, logran una mejor interacción en el sitio activo de la hidrolasa AmiA. 
La ADN Girasa B es una enzima que reduce la tensión de las cadenas de ADN durante su replicación. Los inhibidores atacan el sitio de unión de esta enzima con el ADP. Este nucleótido, debido a los grupos fosfato, es una molécula con carga negativa, que es lo opuesto a la del DRS-SP2, lo que explicaría su baja afinidad.

La diferencia de afinidades del inhibidor con la DRS-SP2 en la proteína de unión de Penicilina tranglicosilasa $1 \mathrm{~b}$, se da por el mismo fenómeno de carga y tamaño, explicado para la proteína de unión de Penicilina G-acil 2A.
La proteasa aspártica secretada posee como inhibidor a la molécula A70. Las proteasas son enzimas que rompen enlaces peptídicos, razón por la cual, la diferencia en la afinidad del inhibidor con esta enzima en comparación con la DRS-SP2 es tan solo 1,6 $\mathrm{kcal} / \mathrm{mol}$, es la menor de todos los casos estudiados. Con esta enzima, la DRS-SP2 no podría ser un inhibidor, ya que los péptidos son sustratos naturales. Estos se van a hidrolizar, por lo que perdería su acción impidiéndoles inhibir la enzima.

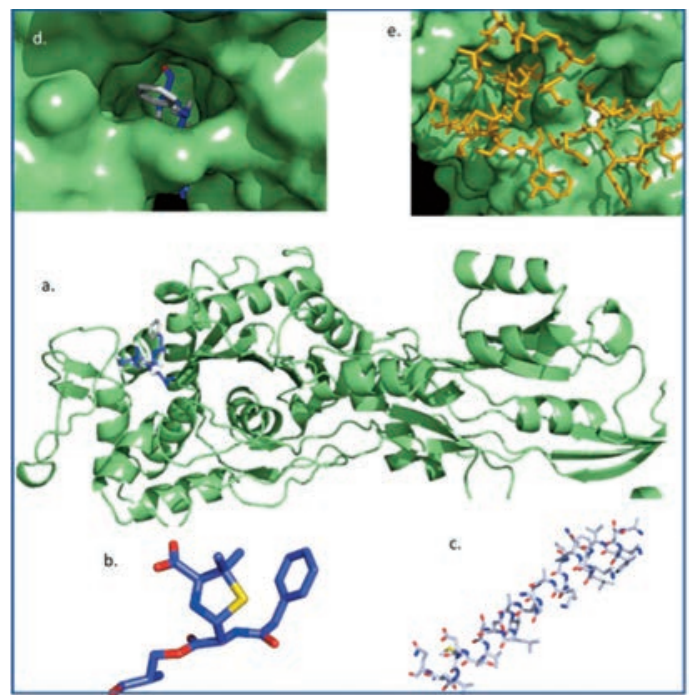

Figura 4. Acoplamiento de la Proteína de unión de Penicilia G-acil 2A. a. Enzima tridimensional. b. Ceftobiprole. c. DRS-SP2. e. Ceftobiprole en el bolsillo enzimático. e. DRS-SP2 en el bolsillo enzimático 
En la interacción de la DRS-SP2 con la membrana celular, más que las energías de interacción, lo que más resalta es cómo el péptido interacciona con la membrana. En el caso de la membrana mamífera, el péptido se mantiene en la superficie, lo que indica que la interacción se da en la superficie y no va a ingresar por la membrana, cosa que sí pasa con la membrana bacteriana. Esto se traduce en baja toxicidad para los seres humanos, ya que este péptido no va a matar células en el cuerpo. Lo contrario va a pasar en la bacteria, donde al penetrar en la membrana va a romperla, causando lisis celular y matando al microorganismo.

\section{CONCLUSIONES}

La DRS-SP2, extraída de Agalychnis spurrelli es un péptido catiónico de cadena corta que posee efectos antimicrobianos sobre bacteria Gram positivas, Gram negativas y hongos. La predicción de sus propiedades muestra un péptido alfa-helicoidal con un punto isoeléctrico de 10,68 y unacarga +3 a pH fisiológico. Estudios de acoplamiento molecular sugirieron que el mecanismo de acción de este péptido no se da por la inhibición de vías enzimáticas vitales para el microorganismo, sino que ataca la membrana celular bacteriana causando lisis celular. Comparación con dermaseptinas de otras especies demostraron que esta secuencia peptídica no se encuentra en ninguna otra especie reportada hasta el día de hoy. Así, esta molécula podría ser estudiada más a fondo como punto de partida para un medicamento antimicrobiano de última generación.

\section{LISTA DE REFERENCIAS}

Ammar, B., Perianin, A., Mor, A., Sarfati, G., Tissot, M., Nicolas, P., Giroud, J. y RochArveiller, M. (1998). Dermaseptin, a Peptide Antibiotic, Stimulates Microbicidal Activities of Polymorphonuclear Leukocytes, Biochemical and Biophysical Research Communications, 247, 870-875. 
Bio-Synthesis. (2018). Peptide Property Calculator, Bio-Synthesis Inc. https://www. biosyn.com/peptidepropertycalculatorlanding.aspx

ChembioDraw (2009). PerkinElmer Informatics

Conlon, J.M. (2012). The Potential of Frog Skin Antimicrobial Peptides for Development into Therapeutically Valuable Anti-Infective Agents. En Rajasekaran, K., Cary, J.W., Jaynes, J.M. y Montesinos, E. (Ed.). Small Wonders: Peptides for Disease Control. Washington, DC: American Chemical Society.

Drozdetskiy, A., Cole, C., Procter, J. y Barton G.J. JPred4: a protein secondary structure prediction server, Nucleic Acids Research, 43, 389-394.

Duellman, W. E. (2001). Hylid Frogs of Middle America. Society for the Study of Amphibians and Reptiles, New York: Ithaca.

Gasteiger E., Hoogland C., Gattiker A., Duvaud S., Wilkins M.R., Appel R.D. y Bairoch A. (2005). Protein Identification and Analysis Tools on the ExPASy Server. En Walker, J. (Ed.). The Proteomics Protocols Handbook, London, Springer

Gill, S.C. y von Hippel, P.H. (1989). Calculation of protein extinction coefficients from amino acid sequence data, Analytical Biochemistry, 182(2), 319-326.

Gray, A.R. (1997). Observations on the biology of Agalychnis spurrelli from the Caribbean lowlands of Costa Rica. Journal of the International Herpetological Society, 22, 61-70.

Guruprasad, K., Reddy, B.V.B. y Pandit, M.W. (1990) Correlation between stability of a protein and its dipeptide composition: a novel approach for predicting in vivo stability of a protein from its primary sequence, Protein Engineering, 4, 155-161.

Holthausen, D., Lee, S, Kumar, V., Bouvier, N., Krammer, F., Ellebedy, A., Wrammert, J., Lowen, A., George, S., Pillai, M. y Jacob, J. (2017). An Amphibian Host Defense Peptide Is Virucidal for Human H1 Hemagglutinin-Bearing Influenza Viruses, Immunity, 46, 587-595. 
Huang L., Chen, D., Wang, L., Lin, C., Ma, C., Xi, X., Chen, T., Shaw, C., y Zhou, M. (2017). Dermaseptin-PH: A Novel Peptide with Antimicrobial and Anticancer Activities from the Skin Secretion of the South American Orange-Legged Leaf Frog, Pithecopus (Phyllomedusa) hypochondrialis, Molecules, 22, 1805.

Innovagen. (2015). Peptide property calculator, Innovagen AB, https://pepcalc.com/

Jones DT. (1999) Protein secondary structure prediction based on position-specific scoring matrices. Journal of Molecular Biology, 292, 195-202.

Lacombe, C., Piesse, C., Sagan, S., Combadière, C., Rosenstein, Y. y Auvynet, C. (2015). Pachymodulin, a New Functional Formyl Peptide Receptor 2 Peptidic Ligand Isolated from Frog Skin Has Janus-like Immunomodulatory Capacities, Journal of Medicinal Chemistry, 58, 1089-1099.

Manzo, G., Casu, M., Rinaldi, M., Montaldo, N., Luganini, A., Gribaudo, G. y Scorciapino, M. (2014). Folded Structure and Insertion Depth of the Frog-Skin Antimicrobial Peptide Esculentin-1b(1-18) in the Presence of Differently Charged Membrane-Mimicking Micelles, Journal of Natural Products, 77(11), 2410-7.

Marani, M., Dourado, F., Quelemes, P., Rodrigues de Araujo, A., Gomes Perfeito, M., Alves Barbosa, E., Costa Véras, L., Rodrigues Coelho, A., Barroso Andrade, E., Eaton, P., Figueiró Longo, J., Bentes Azevedo, R., Delerue-Matos, C. y Leite, J. (2015). Characterization and Biological Activities of Ocellatin Peptides from the Skin Secretion of the Frog Leptodactylus pustulatus, Journal of Natural Products, 78(7):1495-504.

MECN. (2010). Serie Herpetofauna del Ecuador: El Choco Esmeraldeño. Monografía. Museo Ecuatoriano de Ciencias Naturales. Quito, 5:1-232.

Melchiorri, P. y Negri, L. (2009). Amphibian Peptides, Encyclopedia of Neuroscience, San Diego: Elsevier Academic Press.

Nicolas, P. y El Amri, C. (2009). The dermaseptin superfamily: A gene-based combinatorial library of antimicrobial peptides, Biochimica et Biophysica Acta- Biomembranes, 1788(8), 1537-1550. 
Nicolas, P. y Ladram, A. (2013). Dermaseptins. En Kastin, A.J. (Ed.). Handbook of Biologically Active Peptides, Amsterdam: Elsevier.

Ortega-Andrade, H.M. (2008). Agalychnis spurrelli Boulenger (Anura: Hylidae): variación, distribución y sinonimia, Papéis Avulsos de Zoologia, 48(103), 1117.

Samgina, T., Vorontsov, E., Gorshkov, V., Hakalehto, E., Hanninen, O., Zubarev, R. y Lebedev1, A. (2012). Composition and Antimicrobial Activity of the Skin Peptidome of Russian Brown Frog Rana temporaria, Journal of Proteome Research, 11(12), 6213-22.

Scorciapino, M., Manzo, G., Rinaldi, A., Sanna, R., Casu, M., Pantic, J., Lukic, M. y ConIon, J. (2013). Conformational analysis of the frog skin peptide, plasticin-L1 and its effects on the production of proinflammatory cytokines by macrophages, Biochemistry, 52(41), 7231-41.

Stutz, K., Muller, A., Hiss, J., Schneider, P., Blatter, M., Pfeiffer, B., Posselt, G., Kanfer, G., Kornmann, B., Wrede, P., Altmann, K., Wessler, S. y Schneider, G. (2017). Peptide-membrane interaction between targeting and lysis, ACS Chemical Biology, 12(9), 2254-2259.

The PyMOL Molecular Graphics System (2017), Version 2.0 Schrödinger, LLC.

Trott, O. y Olson, A. J. (2010). AutoDock Vina: improving the speed and accuracy of docking with a new scoring function, efficient optimization and multithreading, Journal of Computational Chemistry, 31(2), 455-461.

Wishart, D.S., Feunang, Y.D., Guo, A.C., Lo, E.J., Marcu, A., Grant, J.R., Sajed, T., Johnson, D., Li, C., Sayeeda, Z., Assempour, N., lynkkaran, I., Liu, Y., Maciejewski, A., Gale, N., Wilson, A., Chin, L., Cummings, R., Le, D., Pon, A., Knox, C. y Wilson, M. (2017) DrugBank 5.0: a major update to the DrugBank database for 2018. Nucleic Acids Research, 46, 1074-1082. 